\title{
Colorimetric Accuracy of Color Reproductions in the 3D Scenes
}

\author{
Helena GABRIJELČIČ TOMC, Nika BRATUŽ ${ }^{*}$ Dejana JAVORŠEK
}

\begin{abstract}
Color is a complex phenomenon that depends on the object, the observer and the light source, while the color reproduction additionally includes the surrounding conditions and it depends on the device that can either capture, display or print the reproduction. Colour accuracy is very important for digital reproduction of artistic work where colour represents essence of art. In our research, color reproductions of renderings of computer generated scenes, including an object, background and lights were assessed, and even more, colorimetric accuracy in renderings was analyzed. With assumption that shading in 3D computer generated scenes plays an important role in visual perception of rendered color, the colors were analyzed on renderings of shaded objects. It was determined that colorimetric accuracy depends on the position on rendered objects, either illuminated or shaded. Furthermore, it was discovered that color lightness and hue, light intensity and background influenced $\triangle R G B$ values.
\end{abstract}

Keywords: color accuracy; color rendering; photometric lights; standard lighting type

\section{INTRODUCTION}

One of the most important attributes that define object's appearance is color. Besides directional effects, generated by the direction of the scattered light (i.e. surface phenomena as luster and gloss, shininess and opacity), textures and patterns also influence the human perception of the object's material [1]. Therefore, 3D material modelling consists of the definition of color (spectral variations, defined by the color values of the diffuse map or color coordinates RGB, CIEXYZ, CIELAB), directional effects (scattering and subsurface scattering variations, defined with shading algorithms) and texture (spatial variations of surface represented with small-scale changes in the topology flow building up the object's geometry and maps).

Many researches covered directional effects on the real objects, but on the contrary, only a few papers studied these effects in computer generated graphics and on $3 \mathrm{D}$ objects. Westlund and Meyer demonstrated that with the implementation of Phong, Ward and Cook-Torrance models the directional effects such as gloss, haze and goniometric color can be applied in the computer generated space [2]. The research implemented appearance standards and standard appearance scales that apply user-friendly simulation of mentioned optical phenomena. Pellacini, Ferwerda and Greenberg presented psychophysicallybased light reflection model for image synthesis [3]. Experiments were performed to determine perceptually uniform gloss space and perceptual dimensions of this space. With this methodology, the description of the synthetic generation of surface gloss is possible in computer graphic.

Textures describe spatial variations that could be a part of directional effects or spectral scattering, i.e. atmospheric light scattering effects [1]. Perception of texture in computer graphic was analyzed in a few studies. Heeger and Bergen submitted a method for synthesizing seamless two- and three-dimensional textures from reference input image that is based on human perception of textures [4]. Liu, Dong, Cai, Qi and Chantler have conducted two types of psychophysical experiments (i.e. free grouping and rating) to develop the computational model for generation of procedural textures that consider human perception [5]. They implemented isometric feature mapping to create perceptual texture space that considers the perceptual estimations of the human system. In the research procedural textures were implemented for identification of perceptual dimensions that are used by human perception to distinguish textures. In the results, the perceptual texture space is presented that includes three dimensions, i.e. combinations of perceptual features, used by human visual system. Beside, the results present a training database that can be extended to new procedural models.

Additionally, three basic conditions must be met in the $3 \mathrm{D}$ scene to reach the image synthesis, and consequently final pixel color. These conditions are: the object, the light, and the camera (the virtual eye). The final calculation of the pixel is possible with color transformations and tone mapping. Generation of pixel value occurs according to defined optical characteristics, i.e. materials and textures applied to the mesh geometry as fundamental, enabling redirection of incident light and calculation of reflectance, refraction, scattering and transmission $[6,7]$.

Material and color appearance must be robustly modelled in different illumination and observation conditions of $3 \mathrm{D}$ scene, therefore the radiance should be considered according to the complete spectra. Moreover, all the aspects of color perception should be, as in real world, included and considered in 3D space and 3D rendering. The calculated radiance (computed from material, geometry, texture, directional effects properties) should be a distribution of values of the visible wavelengths and can be achieved with the spectral rendering. This technique enables the transformation from a pixel's spectrum to RGB color space values [6, 7]. This procedure is challenging due to, first, the complex model, in which spectral data are expressed, second, the demanding task to extract significant spectral data from material appearance models and lastly, implemented appearance parameters.

In computer graphic and visualizations, color as perception of visual characteristic was also analyzed in terms of color perception, color constancy, color management and color appearance. Color constancy was studied in the papers by Granzier, Vergne and Gegenfurtner, which proved that material properties such as gloss and matte have significant effects on color constancy [8]. Yang and Maloney demonstrated that the number of illuminants and highlights cues strongly affects 
color constancy, while surface specularity and background cues were discovered to be less important for the perceived color information [9]. Human sensitivity to luminance, chromaticity gradients and other factors in complex 3D scenes were also studied [10,11]. Color management and spectra-based color reproduction algorithm was introduced to spectral reflectance of skin and makeup of 3D avatars by authors Jang, Kim, You and Kim [12]. Goesele, Lensch and Seidel introduced image based BRDF acquisition and color management techniques to high-dynamic range imaging [13]. Studies of color appearance and variations of color parameters analysis, such as chromatic changes [14] and background [15] are well established in analysis of color images, however, only recently Bratuž, Jerman, Gabrijelčič Tomc and Javoršek [16] introduced CIECAM02 color appearance model to computer graphic. Their experiments included 3D scenes with various color and lightness values of objects and background. It was confirmed that CIECAM02 could be also successfully implemented in computer generated space.

In addition, it was also revealed that color reproduction in computer graphic is still an actual focus of interest in many researches that try to improve techniques and methodologies to achieve accuracy in computer generated color appearance [15-18].

The aim of this research was to analyze colorimetric accuracy of rendered colors in a 3D scene with objects illuminated by different light source settings (type of lights and intensity) and rendered on background with different color values. Colour reproduction is very important in $3 \mathrm{D}$ computer generated scenes, yet colorimetric accuracy had not been studied and 3D artists are still using "trial and error" approaches, which are time consuming and depend on subjective evaluation. Implementation of colorimetric accuracy can lead to more controlled colour environment. With the assumption that shading is of a great importance for color appearance and color perception, the phenomenon of colorimetric accuracy was studied on range of colors, bright and shadow positions of 3D objects on different backgrounds rendered with diverse illumination settings. The focus was on deviations between reproductions of colors with different hues, lightness and saturation, so that the analyzed colors could be categorized according to colorimetric accuracy after scene rendering.

\section{METHODS}

\subsection{Scene Setting}

Scene was set up in $3 \mathrm{ds}$ Max in a test chamber and consisted of lights, cameras, and object with shadows (Fig. 1). The scene was illuminated with 4 standard lighting types (abbreviated ST) in the scenes with standard lighting type conditions and with 4 photometric lights (abbreviated $\mathrm{PHO}$ ) in the scenes with photometric illumination conditions. In Fig. 1 only two lights are visible due to side viewport scene representation.

Standard lighting types do not have intensity values that are physically based and are treated as computer-based objects in 3D scene. They simulate different lights from the real world, such as lamps used in houses, offices, streets and, moreover, standard lighting type can also simulate the sun.
Photometric lights are interpreted with light energy by means of properties as standard lighting type, distribution, and color characteristics. These types of lights also cover photometric files used in lightning manufacturing. In our study standard lighting types and photometrical lights had uniform spherical distribution and cast rays of lights in all directions from the single source (types of light: free, omni). Standard lighting type and photometric illumination conditions are presented in Tab. 1.

The positions of the lights and cameras were defined with $x, y$ and $z$ coordinates of the pivot (central) points of these objects. The focus of the cameras was defined with their target, i.e. the position of the camera's focus point (Fig. 1). The positions of the lights were: $x_{\text {light1 }}=-50 \mathrm{~cm}$, $y_{\text {light } 1}=-50 \mathrm{~cm}, x_{\text {light2 }}=50 \mathrm{~cm}, y_{\text {light } 2}=-50 \mathrm{~cm}, x_{\text {light } 3}=-50$ $\mathrm{cm}, y_{\text {light } 3}=50 \mathrm{~cm}, x_{\text {light } 4}=50 \mathrm{~cm}, y_{\text {light } 4}=50 \mathrm{~cm}$, and the height of all lights sources $z_{\text {light1 }}=z_{\text {light2 }}=z_{\text {light3 }}=z_{\text {light4 }}=200$ $\mathrm{cm}$. Two target cameras were positioned in the scene: from top view the position was $x_{\text {cameral }}=0 \mathrm{~cm}, y_{\text {cameral }}=0 \mathrm{~cm}$, $z_{\text {camera } 1}=80 \mathrm{~cm}$, from side view the position was $x_{\text {camera2 }}=$ $50 \mathrm{~cm}, y_{\text {camera } 2}=0 \mathrm{~cm}, z_{\text {camera } 2}=50 \mathrm{~cm}$.

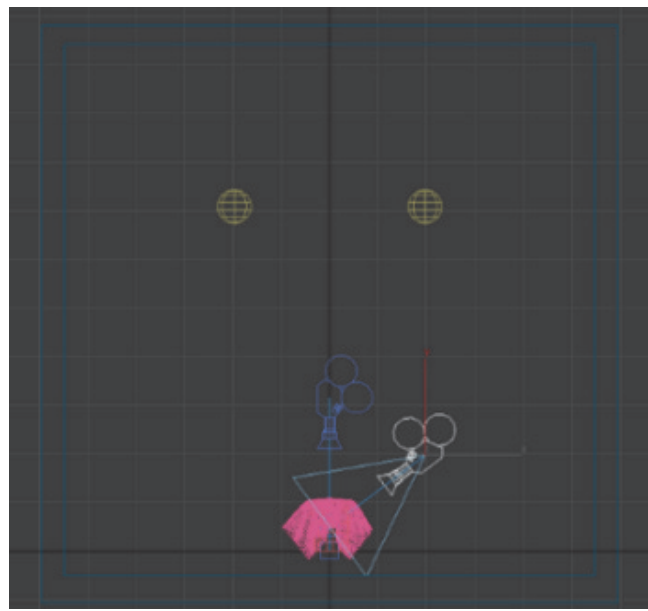

Figure 1 The positions of the lights and cameras (the white camera was used for evaluation)

Optimal conditions for color reproduction (Tab. 1) were defined in preliminary research where colorimetric accuracy of color reproduction of test chart colors illuminated with two types of digital light source and rendered with two rendering engines were analyzed. Namely, standard lighting type (non-photometric) and photometric lights were used, while mental ray was employed to render images. The optimal conditions in dependence of gamma values, illumination and background color were tested, analyzed and defined.

The presented research continues the analysis of issues in the color reproduction in $3 \mathrm{D}$ space with the involvement of a shaded object in the scene where due to topology of the object, there is a formation of shadows on the object and its surrounding. The scenes were rendered in mental ray render engine, i.e. production-quality rendering application based on ray tracing technique.

Light intensity for standard lighting type (internal software light, which is not physically determined) was set to $0.4,0.6,0.8$ and 1 , while light intensity for photometric light with standard illuminant D65 (simulating real world daylight illuminant) was set to 3000 and $4000 \mathrm{~lm}$. Shadows were defined as raytraced. Values of gamma settings and 
defined background are presented in Tab. 1. For standard lighting type other light and rendering settings were default and set to: white color, inverse square decay, no attenuation, directional parameters set to cone and no overshooting, advanced parameters excluded, additional shadow and shadow map excluded, area to render: single frame, full HD, .tiff output file, options and advanced lighting excluded. For photometric lighting type other light and rendering settings were default and set to: white filter color, dimming 1500 candelas, no attenuation, uniform spherical light distribution, in advanced parameters global illumination was checked, additional shadow and shadow map were excluded, area to render was set to single frame, full HD, .tiff output file, custom output size, options excluded and in advanced lighting global illumination was checked.

Table 1 The optimal conditions chosen for testing with mental ray render engine (Bratuž et al., 2015)

\begin{tabular}{|c|c|c|}
\hline \multicolumn{3}{|c|}{ Photometric light } \\
\hline illumination & gamma & background \\
\hline $3000 \mathrm{~lm}$ & $1.8,2.0,2.2$ & $\mathrm{R}=\mathrm{G}=\mathrm{B}=150$ \\
\hline $4000 \mathrm{~lm}$ & $1.8,2.0,2.2$ & $\mathrm{R}=\mathrm{G}=\mathrm{B}=50$ \\
\hline \multicolumn{3}{|c|}{ Standard lighting type } \\
\hline illumination & gamma & background \\
\hline 0.4 & $1.8,2.0,2.2$ & $\mathrm{R}=\mathrm{G}=\mathrm{B}=255$ \\
\hline 0.6 & $1.8,2.0,2.2$ & $\mathrm{R}=\mathrm{G}=\mathrm{B}=200$ \\
\hline 0.8 & $1.8,2.0$ & $\mathrm{R}=\mathrm{G}=\mathrm{B}=100$ \\
\hline 0.8 & 2.2 & $\mathrm{R}=\mathrm{G}=\mathrm{B}=50$ \\
\hline 1 & $1.8,2.0,2.2$ & $\mathrm{R}=\mathrm{G}=\mathrm{B}=0$ \\
\hline
\end{tabular}

\subsection{Selection of Colors}

In our research 15 colors with large and small $\triangle \mathrm{RGB}$ values were chosen from color test chart and tiff texture format was mapped on 3D object. Reference RGB values of chosen color are presented in Tab. 2. Using two shades of for example violet, the influence of colour saturation on $\triangle \mathrm{RGB}$ was analyzed.

Table 2 Reference RGB values

\begin{tabular}{|l|l|l|l|l|l|}
\multicolumn{9}{|c|}{ Table 2 Reference RGB values } \\
\hline patch & R & G & B & color \\
\hline M39 & 157 & 129 & 206 & violet \\
\hline A14 & 155 & 43 & 151 & saturated violet \\
\hline F17 & 31 & 17 & 38 & dark violet \\
\hline I10 & 97 & 191 & 15 & saturated green \\
\hline A39 & 5 & 26 & 15 & dark green \\
\hline A1 & 124 & 141 & 158 & grey \\
\hline H6 & 235 & 239 & 251 & white \\
\hline I18 & & 205 & 2 & 3 & saturated red \\
\hline B27 & & 130 & 32 & 33 & dark red / brown \\
\hline F30 & 130 & 229 & 206 & light cyan \\
\hline A2 & 8 & 16 & 139 & saturated blue \\
\hline N4 & & 231 & 228 & 2 & saturated yellow \\
\hline H23 & & 217 & 165 & 224 & light pink \\
\hline B23 & & 233 & 112 & 205 & pink \\
\hline C1 & & 205 & 5 & 247 & magenta \\
\hline
\end{tabular}

Colors on renderings were analyzed on 13 areas of interest including illuminated parts and shadow parts of draped object (Fig. 2). Bright tones representing a color were detected on areas $2,3,4,7,9,10,13$, mid tones on areas 1, 5, 6, 12 and dark tones on areas 11 and 8. With the consideration of the 3D topology of the object (before rendering), areas analyzed were plane surface 7 (bright part), edges of the fold 2, 4 and 10 (bright parts), inner parts of the fold $1,5,6,8,11,12$ (midtones and shadow parts) and outer parts of the fold 3, 9, 13 (bright parts). Next, to define colorimetric accuracy of renderings, $\triangle \mathrm{RGB}$ color differences were calculated between reference $R G B$ values and rendered RGB values of object colors using the Euclidian distance.

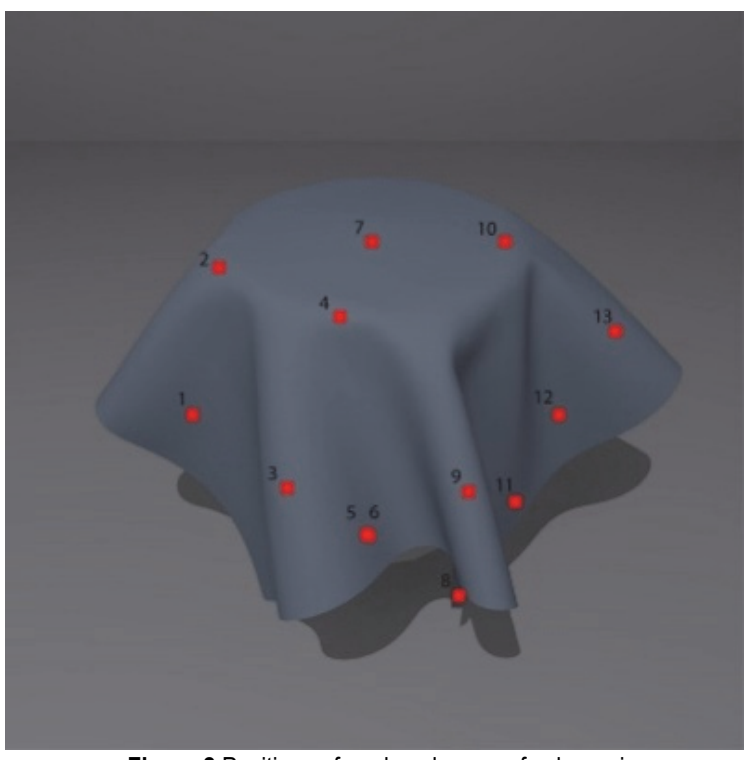

Figure 2 Positions of analyzed areas of color on image

\section{RESULTS AND DISCUSSION}

\subsection{Results of $\triangle R G B$ color difference}

In Figs. 3 to 17, calculated $\Delta \mathrm{RGB}$ color differences are shown. $\triangle \mathrm{RGB}$ color differences were calculated for each color patch, which is described in figure legend with color name (letter and number, e.g. A2), MR stands for mental ray rendering engine, lights type and range (ST for standard lighting type in range of $0.4,0.6,0.8$ and 1 and $\mathrm{PHO}$ for photometric lights set to either 3000 or $4000 \mathrm{~lm}$ ), gamma values set to 2.2 and finally background values ranging from $\mathrm{RGB}=0$ to $\mathrm{RGB}=255$ according to Tab. 1 .

In general, after an observation of results of all colors, light intensities, and background color it is evident that the most shaded positions 8 and 11 have a maximum color difference. Here, the maximum $\triangle \mathrm{RGB}$ was obtained when the intensity was 1 and the standard lighting type was used. These objects' positions are followed by midtones (1, 5, 6 and 12) with lower $\triangle \mathrm{RGB}$ values. The brightest points on the renderings (position 2, 3, 4, 7, 9, 10) have a minimum color difference $\triangle \mathrm{RGB}$. That means that the diffuse shading, which covers an area with a perfectly diffuse reflection of light without specular reflection that brightened color of objects was used. Other positions of tested object had at least some form of shading applied. In most shaded positions dark and midtones (positions 1, 5, 6, 8,11 and 12) are also the most exposed to the phenomena of inter-reflection, i.e. the secondary reflections from the outer parts of the folds, which normals are directed one towards the other. Here, not only formations of shadows (as the positions where there is an absence of direct illumination from light) play an important role but also reflections of selected wavelength influence a color accuracy of the reproductions, which, as mentioned above, have in these positions the highest $\triangle \mathrm{RGB}$ values.

The deeper inspection of results, however, reveals significant deviations of $\triangle R G B$ values depending on object's color, light intensity and background color. 
Highest deviations of $\triangle \mathrm{RGB}$ values were obtained in a case of white color H6, as the color difference at all positions of the image was extremely large (Fig. 8 - white). High $\Delta$ RGB values were also calculated for brighter colors light cyan F30, light pink H23, followed by colors with magenta and pink hues (magenta $\mathrm{C} 1$ and pink B23). In a case of the more saturated and dark colors, smaller $\triangle \mathrm{RGB}$ value between the reference RGB and RGB values of rendered colors was noticed. Examples are black F17 and dark green A39.

Among all parameters, standard lighting type with an intensity of 0.4 , colors gray A1, yellow N4, saturated green I10, violet M39, light cyan F30, saturated red I18, light pink $\mathrm{H} 23$ and pink $\mathrm{B} 23$ had the highest $\triangle \mathrm{RGB}$ values in all positions of tested object except at the position 8 and 11 (shadow parts). This setting was also defined as optimal according to reference [19], based on analysis of color reproduction on testing color chart. Meanwhile, this research is focused on color reproduction of shaded 3D object, therefore it can be presumed that these reproduction settings with the standard lighting type intensity 0.4 and the brightest background color $\mathrm{R}=\mathrm{G}=\mathrm{B}=255$ are not an optimal reproduction conditions for colorimetric accuracy of the colors on shaded 3D object.

In a case of saturated violet A14 $\triangle \mathrm{RGB}$ color difference varies the most according to applied parameters, such as the type and intensity of light. Results of color analysis for the colors magenta $\mathrm{C} 1$, pink B23, light pink $\mathrm{H} 23$ and yellow N4 were very similar as they all reached approximately the same size range of $\triangle R G B$. In case of all mentioned colors, the standard lighting type with light intensity 0.4 had the highest $\triangle \mathrm{RGB}$ (except at the position 8 and 11 , i.e. shadow parts and in a case of magenta), while the other parameters of light intensity showed smallest deviations in $\triangle \mathrm{RGB}$

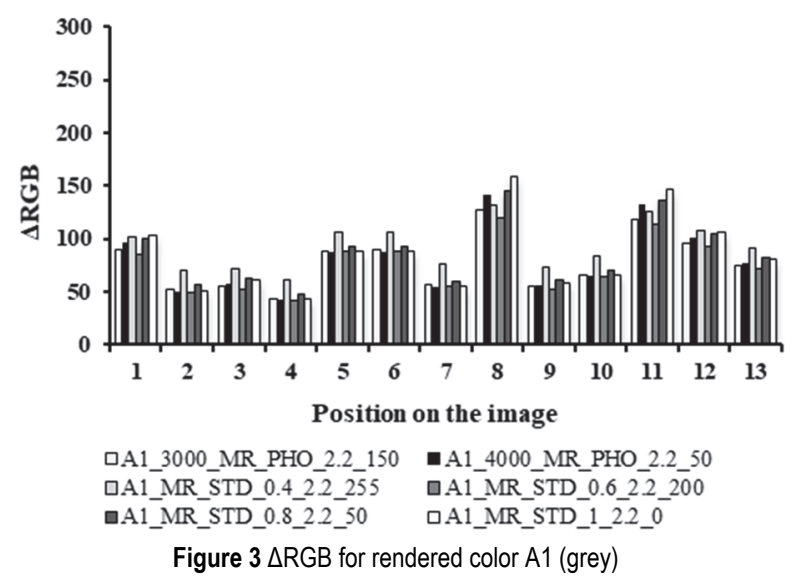

When only photometric illumination is analyzed, it can be found out that light intensity $4000 \mathrm{~lm}$ resulted in higher color differences for all tested hues and saturations of colors in shadow parts of the color reproduction. That was not the case for midtone parts of the colors' reproduction (produced with $4000 \mathrm{~lm}$ of photometric illumination) that had in parts 1 and 12 higher values of color deviations and in parts 5 and 6 generally lower color deviations as reproductions rendered with light intensity $3000 \mathrm{~lm}$. On the contrary, in bright parts $(2,34,7,9,10$ but not 13) of the colors' reproductions, photometric light intensity $3000 \mathrm{~lm}$ had on average higher color deviations than scenes illuminated with $4000 \mathrm{~lm}$. Here, the exceptions were saturated blue color and light pink.
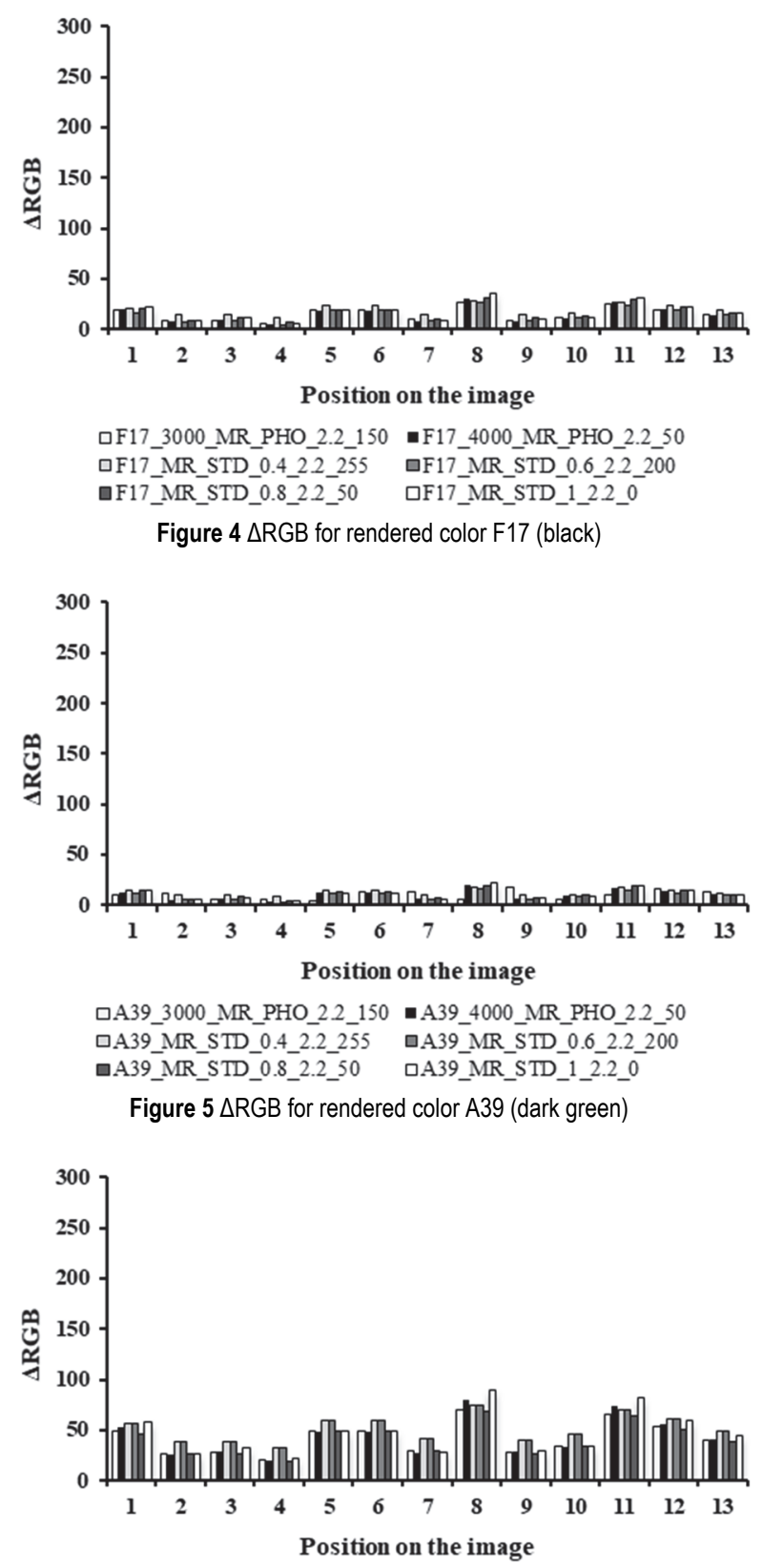

口B27_3000_MR_PHO 22_150 घ B 27 4000_MR PHO 2.250 口B27 MR STD 0.4 22 255 QB27 MR STD 0 6 2.2 200 aB27_MR_STD_0.8_2.2_0 QB27_MR_STD_1_2.2_0

Figure $6 \triangle \mathrm{RGB}$ for rendered color B27 (brown)

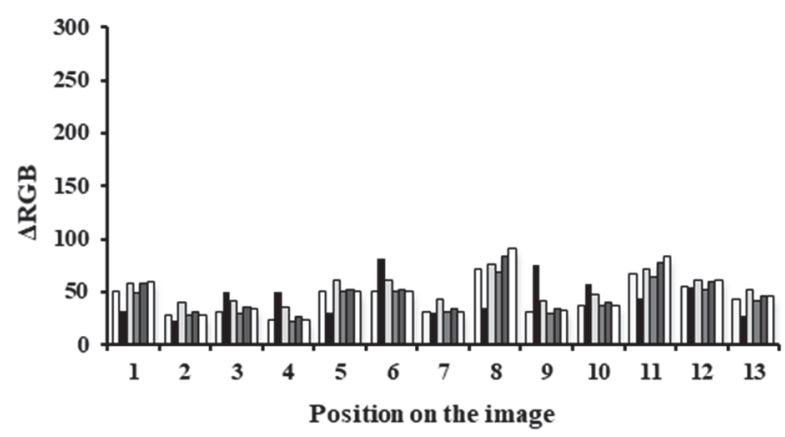

口A2 3000_MR PHO 2.2 150 A 2 4000_MR PHO 2.2 50 口A2_MR_STD_0.4_22_255 पA2_MR_STD_0.6_2.2_200 aA2_MR_STD_0.8_2.250 QA2_MR_STD_1 2.20

Figure $7 \triangle R G B$ for rendered color $A 2$ (sat. blue) 


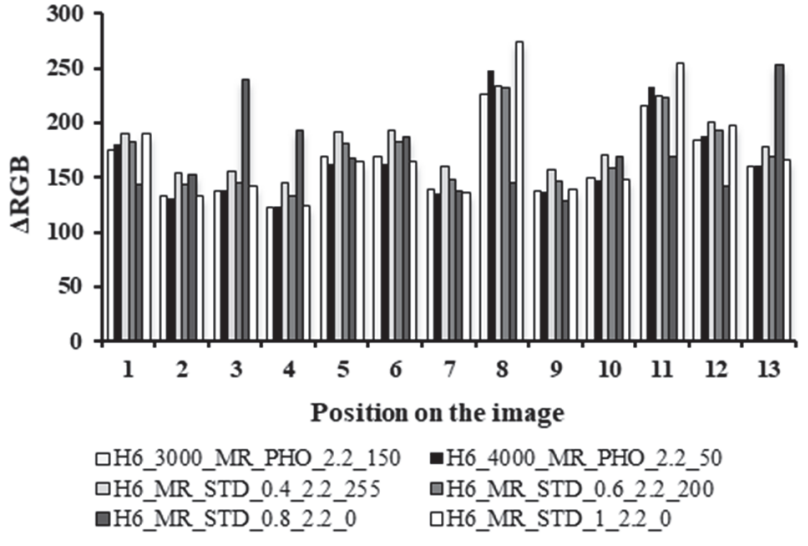

Figure $8 \triangle R G B$ for rendered color $\mathrm{H} 6$ (white)

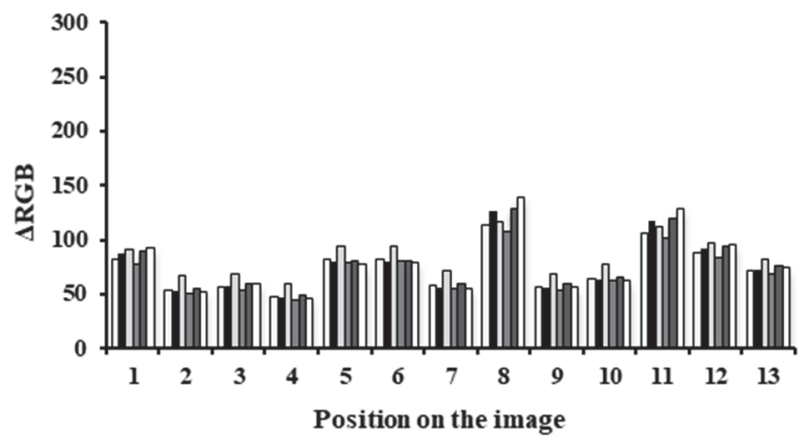

QI10_3000_MR_PHO_2.2_150 - I10_4000_MR_PHO_2.2_50 口I10_MR_STD_0.4_2.2_255 घI10_MR_STD_0.6 2.2_200 aI10_MR_STD_0.8_2.2_50 $\quad$ II10_MR_STD_1_2.2_0

Figure $9 \triangle R G B$ for rendered color 110 (sat. green)

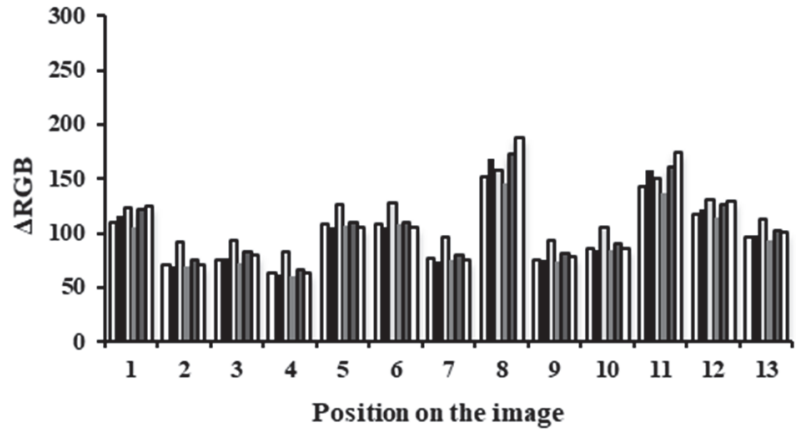

QM39_3000_MR_PHO_2.2_150 - M39_4000_MR_PHO_2.2_50 口M39_MR STD 0.4_2.2 255 M M 39 MR STD_0.6 2.2 200 QM39_MR_STD_0.8_2.2_0 QM39_MR_STD_1_2.2_0

Figure $10 \triangle R G B$ for rendered color M39 (violet)

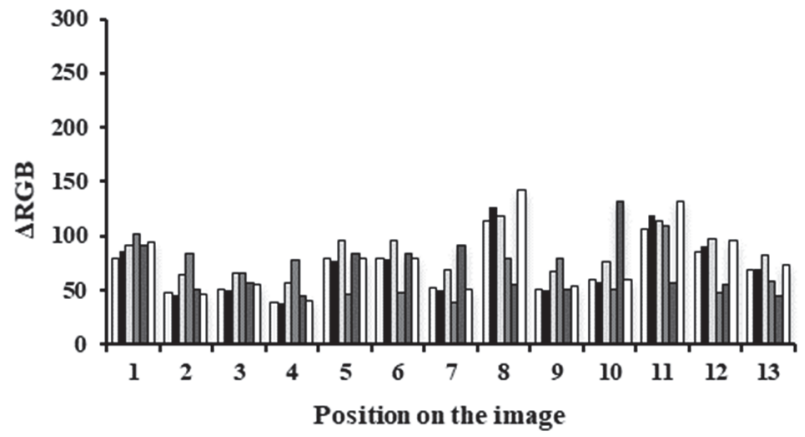

口A14_3000_MR_PHO_22_150 -A14_4000_MR_PHO_2.2_50 aA14_MR STD 0.4 2.2 255 aA14 MR STD 0.62 .2200 -A14_MR_STD_0.8_2.2 50 aA14_MR_STD_1 2.2_0

Figure $11 \triangle R G B$ for rendered color $\mathrm{A} 14$ (sat. violet)

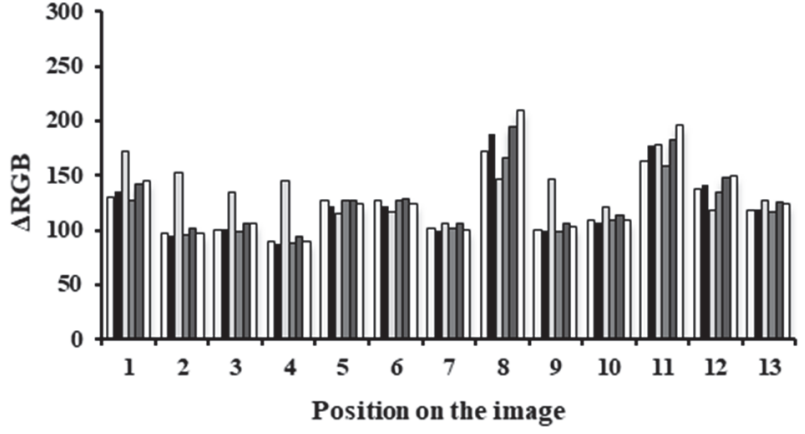

口C1_3000_MR_PHO_22_150 -C1_4000_MR_PHO_22_50 QC1_MR_STD_0.4_2.2_255 aC1_MR_STD_0.6_2.2_200

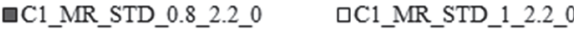

Figure $12 \triangle R G B$ for rendered color $\mathrm{C} 1$ (magenta)

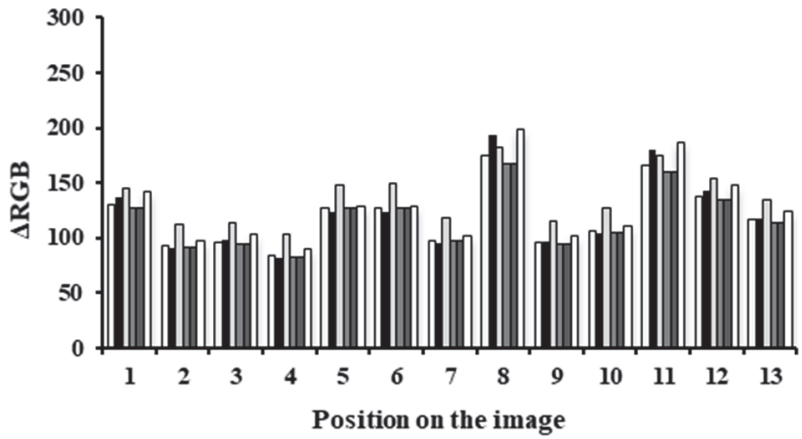

口B23_3000_MR_PHO_2.2150 -B23_4000_MR_PHO_2.2_50 口B23 MR STD 0.4 2.2 255 घB23 MR STD 0.6 2.2 200 QB23_MR_STD_0.8_2.2_0 $\square$ B 23_MR_STD_1 2.2_0

Figure $13 \triangle R G B$ for rendered color $B 23$ (pink)



口H23_3000_MR_PHO_2.2_150 OH23_4000_MR_PHO 2.2 50 口H23 MR STD 0.4 2.2.255 पH23 MR STD 0.6 2.2.200 GH23_MR_STD_0.8_2.2_0 $\square$ H23_MR_STD_1 $2.2 \_0$

Figure $14 \triangle \mathrm{RGB}$ for rendered color $\mathrm{H} 23$ (light pink)

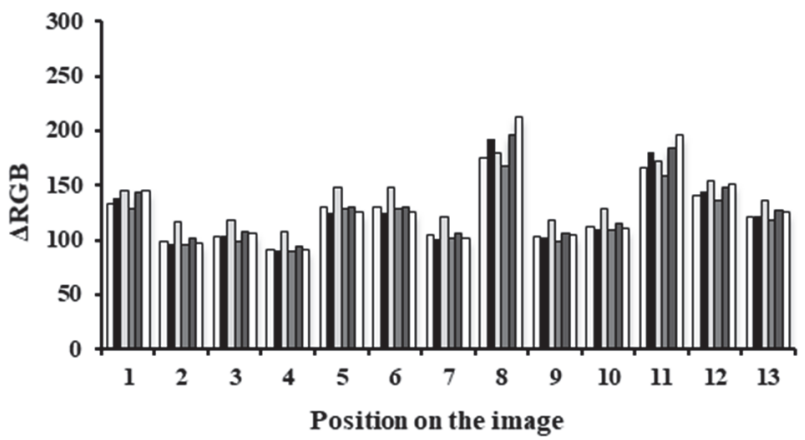

$\square$ N4 3000 MR PHO 2.2 $150 \quad$ N4 4000 MR PHO 2.2 50 口N4 MR STD $0.422255 \quad \square N 4$ MR STD 0.622 .200 aN4_MR_STD_0.8_2.2_0 QN4_MR_STD_1_2.2_0

Figure $15 \triangle R G B$ for rendered color N4 (yellow) 


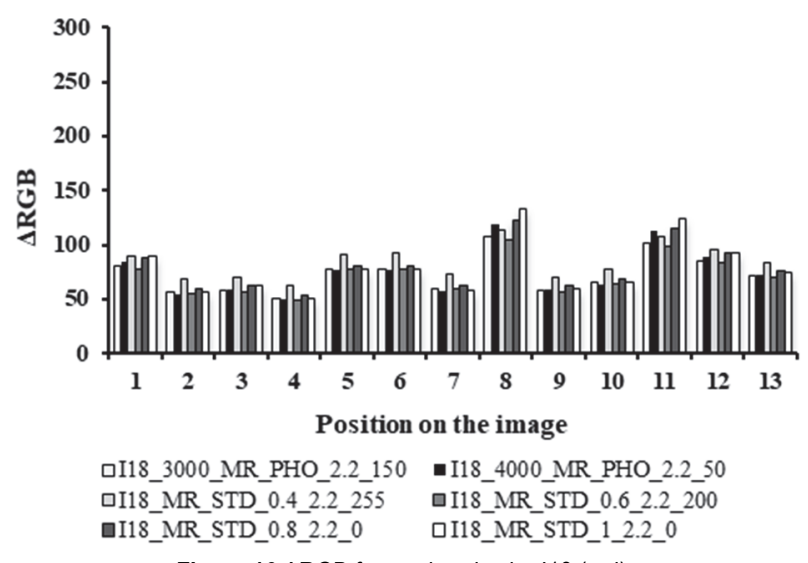

Figure $16 \triangle R G B$ for rendered color 118 (red)



Figure $17 \triangle R G B$ for rendered color $F 30$ (light cyan)

\section{CONCLUSIONS}

In our study we have analyzed whether it is possible to achieve a colorimetrically accurate renderings of colors of shaded object on different background and lightning conditions in the $3 \mathrm{D}$ scene. It was found out that shading significantly influences color reproduction, consequently, exact colorimetric accuracy is not possible since its accuracy depends on color of an object, illumination type and intensity and background color. Comparing color reproductions for objects as whole, highest $\triangle \mathrm{RGB}$ values were obtained for brighter colors, especially for white due to effect of shading. Color differences were high also for other brighter colors included in the analysis, i.e. light cyan, light pink, which reproductions are also influenced on a greater extend by shading. When the reproduction of saturated colors occurs, the color deviations are lower and colors are reproduced more accurately in 3D scene, especially in the examples of saturated blue, violet and green hues. Namely, the reproductions of saturated magenta and pink hues resulted in higher color deviations than reproductions of saturated green, blue and violet. Our research demonstrated also that the accuracy of color reproduction of dark (and saturated) colors is better in comparison with the accuracy of reproductions of brighter colors and colorimetrically accurate rendering of brighter colors is more difficult than the one of darker colors. It was also found out that most shaded positions on the renderings of inspected colors had maximum color difference (minimal colorimetrical accuracy), followed by midtones and bright positions. Among lighting types (standard lightning type, photometric lights) and their intensities the setting of standard lighting type and the intensity 0.4 resulted in the highest color deviations demonstrating that these settings are not optimal for accurate color reproductions. It can be also concluded that photometric illumination and both intensities values (3000 and 4000 $\mathrm{lm})$, depending on shading of the object, demonstrated similar accuracy of color reproduction for different colors included in the analysis.

Method used in this paper can provide a useful tool to assess renderings and colorimetric accuracy in computer generated reproductions of colors and provide a fresh point of view in the field of evaluation of colours and implementation of standardized image analysis in the workflow.

\section{ABBREVIATIONS}

3D

RGB

CIEXYZ

CIELAB

full HD

\section{three dimensional}

color space based on red, green and blue color values

CIE 1931 XYZ color space

CIELAB color space

HDTV high-definition video modes characterized by 1,920 pixels displayed across the screen horizontally and 1,080 pixels down the screen vertically

$\triangle \mathrm{RGB}$ values Euclidian distance between reference RGB values and rendered RGB values

MR mental ray rendering engine

ST standard lighting type

PHO photometric lights

\section{REFERENCES}

[1] Dorsey, J., Rushmeier, H., \& Sillion, F. (2007). Digital modelling material appearance. Burlington, MA: Morgan Kaufmann. https://doi.org/10.1145/1401132.1401140

[2] Westlund, H. B. \& Meyer, G. W. (2001). Applying appearance standards to light reflection models. Proceedings of the 28th annual conference on computer graphic and interactive techniques, 501-551. New York: ACM Press. https://doi.org/10.1145/383259.383318

[3] Pellacini, F., Ferwerda, J. A., \& Greenberg, D. P. (2000). Toward a psychophysically-based light reflection model for image synthesis. Proceedings of the 27th annual conference on computer graphic and interactive techniques, 55-64. New York: ACM Press/Addison-Wesley. https://doi.org/10.1145/344779.344812

[4] Heeger, D. J. \& Bergen, J. R. (1995). Pyramid-based texture analysis/synthesis. Siggraph '95: Proceedngs of the 22nd annual conference on computer graphic and interactive techniques, 229-238. New York: ACM Press. https://doi.org/10.1145/218380.218446

[5] Liu, J., Dong, J., Cai, X., Qi, L., \& Chantler, M. (2015). Visual perception of procedural textures: identifying perceptual dimensions and predicting generation models. PLoS One, 10(6), 1-22. https://doi.org/10.1371/journal.pone.0130335

[6] Pharr, M., Wenzel, J., \& Humphreys, G. (2016). Physically Based Rendering, Third Edition: From Theory to Implementation. Burlington, MA: Morgan Kaufmann.

[7] Dutre, P., Bekaert, P., \& Bala, P. (2006). Advanced Global Illumination, Second Edition. Boka Ranton, FL. https://doi.org/10.1201/b10632

[8] Granzier, J. J. M., Vergne, R., \& Gegenfurtner, K. R. (2014). The effects of surface gloss and roughness on color constancy for real 3-D objects. Journal of Vision, 14(2), 120. https://doi.org/10.1167/14.2.16 
[9] Yang, J. N. \& Maloney, L. T. (2001). Illuminant cues in surface color perception: Tests of three candidate cues. Vision Research, 41, 2581-2600. https://doi.org/10.1016/S0042-6989(01)00143-2

[10] Guzej, A., Hladnik, A., \& Bračko, S. (2016). Examination of Colour Emotions on a Sample of Slovenian Female Population. Tekstile, 9(4), 311-320. https://doi.org/10.14502/Tekstilec2016.59.311-320

[11] Ruppertsberg, A. I., Bloj, M., \& Hurlbert, A. (2008). Sensitivity to luminance and chromaticity gradients in a complex scene. Journal of Vision, 8(9), 1-16. https://doi.org/10.1167/8.9.3

[12] Jang, I. S., Kim, J. W., You, J. Y., \& Kim, J. S. (2013). Spectrum-Based Color Reproduction Algorithm for Makeup Simulation of 3D Facial Avatar. ETRI Journal, 35, 969-979. https://doi.org/10.4218/etrij.13.2013.0079

[13] Goesele, M., Lensch, H. P. A., \& Seidel, H. P. (2004). Validation of Color Managed 3D Appearance Acquisition. Twelfth Color Imaging Conference CIC12, 265-270. Society for Imaging Science and Technology.

[14] Fedorovskaya, A. E., de Ridder, H., \& Blommaert F. J. J. (1997). Chroma variations and perceived quality of color images of natural scenes. Color Research \& Application, 22(2), 96-110. https://doi.org/10.1002/(SICI)1520-6378(199704)22:2<96::AIDCOL5>3.0.CO;2-Z

[15] Trèmeau, A., Nicolas, R., \& Dinet, E. (2008). Influence of Background on the Color Appearance of Images. Journal of Color: Design \& Creativity, 3. Retrieved from: http://www.aic-color.org/journal/v3/jaic_v3_05.pdf

[16] Bratuž, N., Jerman, T., Gabrijelčič Tomc, H., \& Javoršek, D. (2014). Influence of rendering engines on color reproduction. Proceedings of 7th International Symposium in Graphic Engineering and Design, 395-400. Novi Sad, Serbia: Faculty of Technical Sciences.

[17] Xiao, B. \& Brainard D. H. (2008). Surface gloss and color perception of 3D objects. Vis. Neurosci, 25(3), 371-85. https://doi.org/10.1017/S0952523808080267

[18] Morimoto, T., Mizokami, Y., Yaguchi, H., \& Buck, S. L. (2017). Color Constancy in Two-Dimensional and ThreeDimensional Scenes: Effects of Viewing Methods and Surface Texture. Iperception, 8(6), 1-20. https://doi.org/10.1177/2041669517743522

[19] Bratuž, N., Javoršek, D., \& Gabrijelčič Tomc, H. (2015). Defining Optimal Conditions of Colors in 3D Space in Dependence on Gamma Values, Illumination, and background Color. Journal of Imaging Science and Technology, 59(4), 040503-1 - 040503-11. https://doi.org/10.2352/J.ImagingSci.Technol.2015.59.4.040503

\section{Contact information:}

Helena GABRIJELČIČ TOMC, Associate Professor, PhD

Faculty of Natural Sciences and Engineering, Department of Textiles,

Graphic Arts and Design, University of Ljubljana

Snežniška 5, SI-1000 Ljubljana, Slovenia

E-mail: helena.gabrijelcic@ntf.uni-lj.si

Nika BRATUŽ, PhD candidate

(Corresponding author)

Faculty 0 Natural Sciences and Engineering, Department of Textiles,

Graphic Arts and Design, University of Ljubljana,

Snežniša 5, SI-1000 Ljubljana, Slovenia

E-mail: nb8671@student.uni-lj.si

Dejana JAVORŠEK, Associate Professor, PhD

Faculty of Natural Sciences and Engineering, Department of Textiles,

Graphic Arts and Design, University of Ljubljana

Snežniška 5, SI-1000 Ljubljana, Slovenia

E-mail: dejana.javorsek@ntf.uni-lj.si 